\title{
Arduino como elemento notable en prototipos electrónicos
}

\section{Arduino as a notable element in electronic prototypes}

1 Marco Chiluisa-Chiluisa Universidad Central del Ecuador, Quito, Ecuador machiluisa@uce.edu.ec

2 Javier Guaña-Moya

Universidad Central del Ecuador, Quito, Ecuador ejguanam@uce.edu.ec

3 Andrés Carvajal-Proaño https://orcid.org/0000-0002-2329-828X Instituto Tecnológico Superior de Turismo y Patrimonio Yavirac acarvajal@yavirac.edu.ec

4 Rita Paulina Boada-Flores Unidad Educativa Fiscal Numa Pompilio Llona paulina.boada@educacion.gob.ec

Artículo de Investigación Científica y Tecnológica Enviado: 12/11/2021

Revisado: 27/11/2021

Aceptado: 21/12/2021

Publicado:05/01/2022

DOI: https://doi.org/10.33262/concienciadigital.v5i1.1975

Cítese: $\quad$ Chiluisa Chiluisa, M., Guaña Moya, J., Carvajal Proaño, A., \& Boada Flores, R. P. (2022). Arduino como elemento notable en prototipos electrónicos. ConcienciaDigital, 5(1), 104-117. https://doi.org/10.33262/concienciadigital.v5i1.1975

CONCIENCIA DIGITAL, es una Revista Multidisciplinar, Trimestral, que se publicará en soporte electrónico tiene como misión contribuir a la formación de profesionales competentes con visión humanística y crítica que sean capaces de exponer sus resultados investigativos y científicos en la misma medida que se promueva mediante su intervención cambios positivos en la sociedad. https://concienciadigital.org La revista es editada por la Editorial Ciencia Digital (Editorial de prestigio registrada en la Cámara Ecuatoriana de Libro con No de Afiliación 663) www.celibro.org.ec 
Palabras claves:

Tinkercad, arduino, electrónica, tecnología, TIC.
Keywords: Tinkercad, knowledge, electronics, technology, TIC.
Resumen

Introducción. El uso de Arduino en sistemas electrónicos permite articular el hardware y el software de forma amigable e interactiva, motivando al estudiante plasmar su creatividad en el funcionamiento de un circuito eléctrico con la vinculación de elementos electrónicos. Objetivo. Explicar sobre el aprendizaje de Arduino como elemento notable en prototipos electrónicos, en los estudiantes Universitarios de los primeros niveles. Metodología. Se realizó una investigación cuantitativa con los estudiantes, en donde se tomó en cuenta la manera en que es utilizada la plataforma en su educación formativa, también se tomó el Tinkercad como una herramienta digital interactiva, que permite mejorar ciertos aspectos en el campo educativo promoviendo que los contenidos sean llevados a un área digital permitiendo que estos sean más didácticos, entretenidos y de fácil manejo. Resultados. Los resultados se obtuvieron a partir de una encuesta separada por secciones, aplicada a 120 estudiantes, los cuales fueron el 63,3\% hombres y el 36,7\% mujeres. De ellos, el 33\% de los estudiantes afirma que conocen Tinkercad y que algunas veces han manejado esta aplicación online para la electrónica, por otro lado, tenemos un porcentaje muy reducido del $4,2 \%$ de estudiantes que mencionan no conocer este programa y el $25 \%$ describe que casi nunca ha usado dicha aplicación. Conclusión. Las aplicaciones de las TIC, tinkercad, arduino, etc., en las horas clase, permite mejor la iteración entre docentes y estudiantes.

\section{Abstract}

Introduction. The use of Arduino in electronic systems allows the articulation of hardware and software in a friendly and interactive way, motivating the student to express their creativity in the operation of an electrical circuit with the linking of electronic elements. Objective. Explain about the learning of Arduino as a remarkable element in electronic prototypes, in the University students of the first levels. Methodology. Quantitative research was carried out with the students, where the way in which the platform is used in their formative education was considered, Tinkercad was also taken as an interactive digital tool, which allows improving certain aspects in the educational field by promoting that the contents are taken to a digital area allowing them to be more didactic, entertaining and easy to use. Results. The results were 
obtained from a survey separated by sections, applied to 120 students, who were $63.3 \%$ men and $36.7 \%$ women. Of them, $33 \%$ of the students affirm that they know Tinkercad and that they have sometimes handled this online application for electronics, on the other hand, we have a very small percentage of $4.2 \%$ of students who mention not knowing this program and the $25 \%$ describe that they have hardly ever used such an application. Conclusion. ICT applications, tinkercad, arduino, etc., in class hours, allow better iteration between teachers and students.

\section{Introducción}

El presente artículo tiene como objetivo conocer la utilización de Arduino como elemento notable en prototipos electrónicos, en los estudiantes de la Carrera de Informática, de la Facultad de Filosofía, Letras y Ciencias de la Educación. Las TIC dentro de la formación educativa posiblemente han tomado mayor relevancia en los últimos años, por lo cual se pretende acceder a conceptos y teorías a través de una investigación analítica (Luna et al., 2018).

De acuerdo con Naranjo-Villota et al. (2020), la accesibilidad a las distintas herramientas tecnológicas se convierte en una estrategia que los docentes emplean en el proceso de enseñanza-aprendizaje, y que a su vez permiten al estudiante verificar los resultados obtenidos dentro del aula. Ahora bien, existen diferentes formas de realizar simulaciones y prototipos eléctricos, para lo cual se recomienda la revisión de información verídica propuesta en: libros, videos educativos, portales didácticos, entre otros, los mismos que están disponibles en la nube.

Pérez-Fabara et al. (2017), describe que las TIC son herramientas que ayudan a la dinamización de las metodologías docentes en beneficio del estudiante. La finalidad de emplearlas es proporcionar aprendizajes significativos, desarrollar habilidades y comportamientos, de una manera eficiente y eficaz en un periodo corto de tiempo (Moya et al., 2016).

El presente artículo describe las normas y parámetro de Arduino empleado en la plataforma virtual Tinkercad, por lo cual Ardila y Duban (2021), describen que estas herramientas se utilizan para la programación y simulación de circuitos y objetos 3D. Durante el proceso evaluativo de los aprendizajes, se observa que los instrumentos de evaluación han evolucionado con el desarrollo de la sociedad y las nuevas tecnologías. Los resultados educativos, anteriormente, se obtenían a través de instrumentos físicos que no mostraba el $100 \%$ de las destrezas de los estudiantes. Actualmente, la mayoría de las 
docentes promueve las TIC como estrategia metodológica empleando una gama de herramientas virtuales para lograr el aprendizaje significativo en el estudiantado (Ordóñez et al., 2020).

El propósito de la investigación es conocer la percepción del uso de Arduino y Tinkercad en el diseño de circuitos electrónicos, para su construcción en el ámbito académico; tomando en cuenta que el sistema educativo ha tenido un leve descenso de calidad en los últimos años y ha generado un cambio social (Esteve, 2006). Por tal razón, es necesario implementar estas herramientas para elevar el nivel educativo actual. Además, se pretende que las plataformas digitales compartan la dicotomía estrategia metodológicainstrumentos evaluativo del proceso enseñanza-aprendizaje.

\section{Arduino}

Arduino es considerada una plataforma de creación electrónica de código abierto, posee una placa de circuito impreso que funge como microcontrolador, de la marca "ATMAEL", cuenta con entrada y salida (analógicas y digitales), en un entorno de desarrollo que está basado en el lenguaje de programación processing. El dispositivo conecta el mundo físico con el mundo virtual, a través de sensores y actuadores controla alarmas, sistemas de luces, motores, sistemas comunicaciones, entre otros (Ruiz, 2017).

En el mercado existen diferentes microcontroladores y plataformas para programar, sin embargo, las funcionalidades y herramientas de la mayoría poseen un grado elevado de complejidad en su manejo. Arduino, por su parte, ofrece a sus usuarios una serie de ventajas e idóneas características en perspectiva con otros sistemas (Tapia-Ayala \& Manzano-Yupa, 2013).

Los aspectos que se pueden resaltar de Arduino son los siguientes:

- Factibilidad: La placas Arduino son más accesibles debido a que los costes de los elementos necesarios para su funcionamiento no son levados, comparados con otras plataformas y microcontroladores.

- Multi-Plataforma: El software de Arduino funciona en los sistemas operativos Windows, Macintosh OSX y Linux. Por otra parte, la mayoría de los entornos para microcontroladores están limitados a Windows.

- Ambiente de programación sencillo y directo: El ambiente de programación de Arduino es fácil de usar para los usuarios, especialmente para lo que están iniciando. Arduino está basado en el entorno processing con lo que el usuario aprenderá a programar desde cero y podrá irse familiarizando con los lenguajes de programación.

- Software ampliable y de código abierto: El software Arduino está publicado bajo una licencia libre, además puede ser ampliado por los programadores y 
desarrolladores experimentados. El lenguaje puede extenderse a través de librerías de $\mathrm{C}++$ y modificarse empleando un lenguaje de programación AVR C.

- Hardware ampliable y de código abierto: Arduino está basado en los microcontroladores ATMEGA168, ATMEGA328 y ATMEGA1280. Los planos de los módulos están publicados bajo licencia creative commons, por lo que los diseñadores de circuitos pueden hacer su propia versión de módulo, ampliándolo u optimizándolo, facilitando el ahorro.

\section{Tinkercad}

Tinkercad es una aplicación gratuita y en línea especializada en el diseño e impresión 3D, de propiedad de Autodesk, la cual permite realizar simulaciones en tiempo real y programación de dispositivos Arduino virtuales. Los sketches se pueden elaborar utilizando textos o bloques (de forma similar a la aplicación Scratch), la cual también es una herramienta que permite exportar el circuito a diagrama de pistas (PCB) el cual es compatible con Eagle (también propiedad de Autodesk) (Ramos-Venegas, 2019).

\section{Uso del simulador de Arduino en Tinkercad}

Antes de realizar un circuito real, es posible probar una simulación como manera de asegurar que todo funciona correctamente. Para esto, existen herramientas gratuitas como la ofrecida por Autodesk, disponible en la página “https://tinkercad.com/". A continuación, se muestran los pasos para elaborar un circuito básico con esta herramienta.

1. Registrarse y acceder a la opción "Circuitos - Crear Nuevo Circuito".

2. Se desplegará un proyecto nuevo donde se debe empezar a introducir componentes.

3. Existen diferentes opciones dentro del circuito. Algunas de las más importantes son:

- Para editar el código se debe seleccionar "editar código"

- Se puede añadir componentes de la librería, si se selecciona la opción "+Componentes"

- Para comenzar la simulación seleccionar la opción "Iniciar Simulación"

4. Si selecciona la opción "+Componentes" en la parte inferior aparecerá un desplegable con varias opciones disponibles. La principal es la placa Arduino Uno, dentro de la subcarpeta Arduino Basic Kit.

5. Para introducir un componente en el circuito pinchar una vez en él y otra sobre el esquema, en la posición deseada. Se puede eliminar el componente pulsando sobre este y seleccionando "Suprimir o Retroceso". Los componentes con pines se pueden interconectar mediante cables, para eso hay que pinchar sobre un pin y después pinchar en otro pin para cerrar la conexión. Los elementos introducidos se pueden editar de manera muy fácil. 
6. Para modificar el código, pulsar sobre "Code Editor", con lo cual se desactivará el botón "Block" brindando acceso al editor.

7. Una vez introducido el código correspondiente se puede simular la ejecución real del circuito seleccionando la opción "Start Simulation".

\section{Trabajos relacionados}

En un estudio presentado en el año 2019, se implementaron las tarjetas Arduino para el desarrollo de una variedad de prototipos diseñados para brindar soporte en los procesos de educación y asistencia, también para generar una revolución en estos entornos. Los resultados fueron alentadores pues mejoraron tanto las condiciones de trabajo de educadores, doctores, etc., como las condiciones de vida de los estudiantes, pacientes y familiares. Esto abrió nuevas posibilidades para que las personas crezcan y se desenvuelvan en la sociedad moderna. La programación de componentes dentro del ambiente de Arduino, puede ser una tarea larga y complicada, sobre todo al momento de iniciar la estructura individual del código de cada componente, haciendo que la programación limite a los estudiantes que no tienen conocimiento sólido sobre este tema (Chiluisa \& Ortegas, 2019).

En el año 2013 una investigación realizó una propuesta que aporta al conocimiento y experiencia para la implementación de la tesis, donde se realizaron pruebas de adquisición de datos con respecto a Labview con Arduino. En la práctica se comprobó que el sensor MTS 360, cumplió las condiciones del diseño y por ende se realizaron pruebas en la programación del software Arduino (Tapia-Ayala \& Manzano-Yupa, 2013).

Un estudio presentado en el año 2018 mostró los resultados preliminares de un análisis sobre la evaluación de aprendizajes en los estudiantes de informática administrativa, donde la incorporación de recursos tecnológicos en el proceso de enseñanza y aprendizajes es más frecuente que la presentación y realización de tareas o actividades manuales. Los autores se enfocan en la E-evaluación formativa, a partir de esto se obtuvieron dos posturas sobre el uso de herramientas tecnológicas: la primera es que, según los docentes, agilizan el proceso y permiten identificar cuáles son los temas para reforzar en clases, y la segunda propone que los estudiantes pueden identificar de manera automática su avance y la calificación de la actividad o tarea (Castillo et al., 2018).

Una investigación, realizada en el año 2018, puso en práctica una propuesta metodológica para desarrollar el proceso evaluativo utilizando las TIC. Básicamente se realizó para poder evaluar de forma precisa el aprendizaje continuo de los alumnos, obteniendo de esta evaluación calificaciones cuantitativas, y a su vez dar seguimiento académico a cada alumno para poder aplicar la retroalimentación. Se concluye que, las herramientas gratuitas online actúan como un sistema de respuesta inteligente, con el que los docentes pueden generar varios tipos de cuestionarios que brinden respuestas al instante y puedan 
rendirse desde un dispositivo reduciendo de esta manera el riesgo de copia (Reyes \& Sahuquillo, 2018).

En el año 2018 se desarrolló una investigación sobre el uso de los sistemas de respuesta interactiva como herramienta para favorecer el aprendizaje proactivo. Este proyecto ofrece soluciones para las falencias de la formación a distancia, pero estas también sirven de apoyo en el sistema educativo presencial. En este estudio se ha implementado una nueva metodología de trabajo en laboratorio, fomentando el aprendizaje proactivo del alumno, con el objetivo de dinamizar las clases. El estudio previo fuera del aula se ha cumplido, observándose una mayor motivación e innovación en el proceso de aprendizaje (Perea-Moreno, 2018).

En un estudio del año 2018 se evidenció como la evaluación y las TIC'S de software libre mejoraron el rendimiento académico en una unidad educativa en el Azuay. Los resultados señalan que el uso de la tecnología en la actualidad es casi imprescindible en todos los campos de la ciencia, siendo las herramientas y servicios de la informática parte de los recursos que profesionales de todas las áreas deben tener en cuenta al momento de realizar sus tareas. Proponiendo un proyecto educativo con énfasis en software libre, mediante la guía práctica y aprendiendo de la misma fue realizada la investigación exitosamente en la unidad educativa fiscal "Provincia de Azuay" (Ibarra, 2018).

\section{Metodología}

La metodología de la presente investigación corresponde a un enfoque mixto. En una primera instancia se recolectó datos cualitativos pues se empleó el análisis documental para teorización de las variables. Además, por medio de la revisión bibliográfica, se ha seleccionado los estudios relevantes llevando a cabo un análisis descriptivo de contenido. El segundo momento de la investigación corresponde a la recolección de datos cuantitativos utilizando la técnica de la encuesta, con el cuestionario como instrumento, para reforzar y verificar los resultados de este artículo.

En relación con el procedimiento de investigación, se desarrollaron las siguientes fases:

La recolección y análisis de las fuentes documentales se emplearon como medio para la descripción y caracterización de las variables, como son Las TIC, Arduino y Tinkercad.

La elaboración del instrumento para la recolección de datos cuantitativos se desarrolló en base a las dimensiones conceptualizadas y caracterizadas por la investigación bibliográfica documental. El objetivo de las encuestas fue obtener el criterio de los estudiantes, de la carrera de Pedagogía de las Ciencias Experimentales mención Informática, que pertenece a la Facultad de Filosofía, Letras y Ciencias de la Educación de la Universidad Central del Ecuador, respecto al contenido e implementación de 
Arduino y Tinkercad como herramientas metodológicas para la dinamización de las clases.

La evaluación de Tinkercad como herramienta para programar, fue por parte de los estudiantes de la Carrera de Informática, debido a que estos poseen las bases sobre lenguaje de programación. Debido a la pandemia, la encuesta fue virtual, lo cual facilitó la obtención y tabulación de resultados.

Los resultados que arrojó la investigación se presentarán utilizando tablas y figuras, las cuales permitirán una mejor comprensión de los datos, por parte del lector, y un análisis sustancioso. La criticidad de los estudiantes permitió desarrollar una exploración participativa con la obtención exitosa de los resultados requeridos.

\section{Resultados}

Los resultados se obtuvieron a partir de una encuesta separada por secciones, aplicada a 120 estudiantes de la carrera de pedagogía de las ciencias experimentales informática, de la facultad de filosofía letras y ciencias de la educación, de la Universidad Central del Ecuador, para conocer el uso y conocimiento que poseen acerca del programa en online Tinkercad.

En la primera sección se encuentra el apartado de sexo, para poder determinar la cantidad de hombres y mujeres que realizaron esta encuesta. Lo cual nos permite conocer el porcentaje en relación con el total de estos. Los cuales se detallan en la tabla 1 que se encuentra a continuación.

Tabla 1

Datos de la muestra

\begin{tabular}{ccc}
\hline Criterios & Respuestas & Porcentaje \\
\hline Hombres & 76 & $63.30 \%$ \\
Mujeres & 44 & $36.70 \%$ \\
Total & 120 & $100.00 \%$ \\
\hline
\end{tabular}

Los datos de la muestra que nos arrogó la encuesta hacen referencia a la cantidad de hombres y mujeres que la realizaron. Teniendo así un total de 76 hombres que corresponden al $63,30 \%$ de los estudiantes encuestados, mientras que un total de 44 mujeres que corresponden $36,70 \%$ de mujeres encuestadas.

En la tabla 2 se hace referencia al nivel de conocimiento o acercamiento que tienen los estudiantes con las placas de Arduino teniendo, como criterios a responder por el estudiantado, nunca, casi nunca, a veces, casi siempre, siempre. De esta forma se facilita 
la obtención de respuestas para convertirlas en porcentaje y validar en relación con el porcentaje total.

\section{Tabla 2}

Conocimiento de Arduino

\begin{tabular}{lll}
\hline Criterios & Respuestas & Porcentaje \\
\hline nunca & 20 & $16,70 \%$ \\
casi nunca & 15 & $12,50 \%$ \\
a veces & 30 & $25 \%$ \\
casi siempre & 25 & $20,80 \%$ \\
siempre & 30 & $25 \%$ \\
\hline Total & 120 & $100 \%$ \\
\hline
\end{tabular}

Según los resultados obtenidos podemos destacar que el mayor porcentaje de respuesta en relación con los criterios ponderados es de $25 \%$ de los estudiantes que conocen Arduino o han manejado esta herramienta para la electrónica, por otro lado, un $16.7 \%$ menciona no saber que son los Arduino.

La tabla 3 corresponde al indicador que hace referencia al conocimiento que tienen los estudiantes encuestados en el programa en online Tinkercad. Los criterios para responder son nunca, casi nunca, a veces, casi siempre y siempre. De esta forma se facilita la obtención de respuestas para convertirlas en porcentaje y validar en relación con el porcentaje total.

Tabla 3

Conocimiento de Tinkercad

\begin{tabular}{|c|c|c|}
\hline Criterio & Respuestas & Porcentaje \\
\hline nunca & 20 & $16,70 \%$ \\
\hline casi nunca & 20 & $16,70 \%$ \\
\hline a veces & 35 & $25 \%$ \\
\hline casi siempre & 15 & $12,5 \%$ \\
\hline siempre & 30 & $25 \%$ \\
\hline Total & 120 & $100 \%$ \\
\hline
\end{tabular}

Según los resultados obtenidos podemos destacar que el mayor porcentaje de respuesta en relación con los criterios ponderados es de 33\% de los estudiantes que no conocen Tinkercad o han manejado este programa online para la electrónica, por otro lado, 
tenemos un porcentaje muy reducido pues el 4,2\% de estudiantes mencionan conocer este programa.

La tabla 4 corresponde al indicador que hace referencia al uso de las placas de Arduino, por parte de los estudiantes, para el proceso educativo. Los criterios para responder son nunca, casi nunca, a veces, casi siempre y siempre. De esta forma se facilita la obtención de respuestas para convertirlas en porcentaje y validar en relación con el porcentaje total.

\section{Tabla 4}

Uso de Arduino en proceso educativo

\begin{tabular}{ccc}
\hline Criterio & Respuestas & Porcentaje \\
\hline nunca & 5 & $4,20 \%$ \\
casi nunca & 30 & $25,00 \%$ \\
a veces & 40 & $33 \%$ \\
casi siempre & 35 & $29,2 \%$ \\
siempre & 10 & $8 \%$ \\
\hline Total & 120 & $100 \%$ \\
\hline
\end{tabular}

Según los resultados obtenidos se puede destacar que el mayor porcentaje, 33\%, de las respuestas se encuentra relacionado con nunca. Mientras que un porcentaje menor, $4,2 \%$, se encuentra relacionado con siempre que hace referencia al poco porcentaje de estudiantes que usan Arduino en su proceso educativo.

La tabla 5 corresponde al indicador que hace referencia a la enseñanza de Arduino y Tinkercad en la carrera de pedagogía de las ciencias experimentales informática y los conocimientos que tienen los estudiantes encuestados en el programa en online Tinkercad. Los criterios para responder son nunca, casi nunca, a veces, casi siempre y siempre. De esta forma se facilita la obtención de respuestas para convertirlas en porcentaje y validar en relación con el porcentaje total.

\section{Tabla 5}

Enseñanza de Arduino y Tinkerd por parte de la Carrera

\begin{tabular}{lll}
\hline Criterios & Respuestas & Porcentaje \\
\hline nunca & 40 & $33,00 \%$ \\
casi nunca & 30 & $25,00 \%$ \\
a veces & 25 & $20,8 \%$ \\
casi siempre & 20 & $16,7 \%$ \\
siempre & 5 & $4,2 \%$ \\
\hline Total & 120 & $100 \%$ \\
\hline
\end{tabular}


Según los resultados obtenidos se puede destacar que el mayor porcentaje de las respuestas se encuentra relacionado con nunca (33\%). Mientras que un porcentaje mucho menor se encuentra relacionado con siempre $(4,2 \%)$ que hace referencia al poco porcentaje de estudiantes que mencionan la consideran que ha existido enseñanza de Arduino y Tinkercad en su proceso educativo dentro de los salones de clase.

\section{Conclusiones}

- Actualmente casi nadie duda de que hay que impartir nociones de programación y robótica en las clases de tecnología, y hay mucho interés además por la conexión que esto tiene con las STEM/STEAM, tan de moda hoy en día (Science, Technology, Engineering, Arts, Mathematics) y en este ambiente Arduino una de las herramientas más adecuadas, por su bajo costo, sencillez, posibilidades de expansión y soporte de la comunidad.

- Es por esto por lo que definitivamente los docentes estamos llamados al reto de encontrar las herramientas que más se acerquen al sentir de los estudiantes modernos, que los motiven a partir de sus capacidades, para que sean partícipes activos del proceso enseñanza aprendizaje. Como se ha exhibido en este trabajo, la inclusión de las herramientas de las TIC en la educación de las ingenierías supone un atractivo especial y conlleva a mejorar los resultados de aprendizaje en los estudiantes.

- El Arduino surge como una plataforma ideal como complemento e incluso como eje central en algunos casos para que los estudiantes desarrollen el conocimiento de forma participativa y constructiva. Además, apliquen desde la práctica los conocimientos básicos teóricos de manera que puedan acceder a un aprendizaje profundo, mediante el acercamiento a modelos de aplicación basados en experiencias similares a las encontradas en las industrias del mundo, pero con la ventaja de usar materiales de bajo costo, de código abierto y de fácil implementación.

- Se destaca que el porcentaje de estudiantes que mencionan desconocer acerca de los temas relacionados con las placas de Arduino y el programa Tinkercad es alto, por lo que se debe tomar en cuenta estas menciones para enfatizar en el proceso de enseñanza de la materia de Electricidad dentro de la carrera de Informática, así de esta forma hacer uso de estas herramientas para que el alumnado adquiera aprendizajes significativos que influyan en su proceso de enseñanza-aprendizaje.

- El estudiantado desconoce acerca de las bondades que brinda el programa Tinkercad, ya que al ser un programa en 3D online brinda la posibilidad de experimentar diferentes acciones correspondientes a la programación en Arduino y sus conexiones, por lo que es necesario inmiscuir al estudiantado con estas herramientas para que de esta forma pueda hacer uso de estas para el refuerzo de 
su aprendizaje como actividades sincrónicas o asincrónicas dentro de las horas clase o fuera de ellas.

\section{Referencias bibliográficas}

Ardila Galindo \& Duban Armando. (2021). Estrategia Didáctica para Abordar Circuitos Eléctricos a través de la Plataforma Tinkercad para Desarrollar las Competencias Tecnológicas en los Estudiantes de Grado $6^{\circ}$ del CUAN Usme.

Castillo-Mendoza, Cotera-Regalado \& Soberanes-Martín. (2018). E-Evaluación del aprendizaje de los Estudiantes de Informática Administrativa. Pista Educativa, No. 130, noviembre 2018, México, Tecnológico Nacional de México en Celaya.

Chiluisa-Castillo \& Ortegas-Barreto (2019). Herramienta inteligente web de soporte en la generación de prototipos electrónicos para el desarrollo de proyectos de inclusión educativa. Proyecto Técnico Obtenido de https://dspace.ups.edu.ec/bitstream/123456789/17501/1/UPS-CT008347.pdf

Esteve, J. M. (2006). El sistema educativo ante la encrucijada del cambio social: Una mirada hacia el futuro. Universidad de Málaga.

Ruiz Gutiérrez, J. M. (2017). Manual de Programación Arduino. Transl.: BW Evans et al., Arduino Notebook: A Beginner's Reference. Obtenido de https://arduinobot.pbworks.com/f/Manual+Programacion+Arduino.pdf

Ibarra, Mora, L. N. (2018). Influencia de las TIC's de software libre en la calidad del rendimiento académico en la asignatura de lengua y literatura, en los estudiantes de octavo año de educación general básica de la Unidad Educativa Provincia del Azuay período lectivo 2015 - 2016. Obtenido de http://repositorio.ug.edu.ec/handle/redug/27975

Luna-Echeverría, N., Erazo-Luna, A., Huebla-Huebla, F. \& Guaña-Moya, J. (2018). Uso de las Tecnológicas de la Información y Comunicación (TIC) en los Institutos de Educación Superior. Polo del Conocimiento, 3(10), 300-315.

Moya, E. J. G., Herrera, D. G. G. \& Arequipa, E. E. Q. (2016). Utopía o realidad de aplicaciones informáticas en la educación. Caso Universidad Ecuatoriana. Revista Publicando, 3(9), 119-137.

Naranjo-Villota, D., Guaña-Moya, J., Acosta-Vargas, P., \& Muirragui-Irrazábal, V. (2020). Evaluación de la accesibilidad web en institutos acreditados de educación superior del Ecuador. Revista Espacios, 41(04). 
Ordóñez-Almeida, K., Guaña-Moya, J., García-Herrera, D., Naranjo-Villota, D., BonillaMorales, C. \& Cajamarca-Yunga, J. (2020). Análisis del uso de los recursos en la plataforma virtual de enseñanza aprendizaje. Revista Ibérica de Sistemas e Tecnologias de Informação, (E32), 126-136.

Perea-Moreno, A. (2018). El uso de los sistemas de respuesta interactiva como herramienta para favorecer el aprendizaje proactivo en ingeniería.

Pérez-Fabara, M. A., del Carmen Rojas-Arias, R., Quinatoa-Arequipa, E. E., \& Moya, E. J. G. (2017). Las tecnologías en el mejoramiento de los procesos educativos en la Educación Superior en América Latina. Revista Publicando, $4(11$ (1)), 704-718.

Reyes, M. \& Sahuquillo, O. (2018). Propuesta de una metodología de evaluación del aprendizaje basada en las TIC. Universidad Politécnica de valencia. Obtenido de: http://dx.doi.org/10.4995/INRED2018.2018.8622

Ramos-Venegas, J. (2019). Introducción a Tinkercad. Obtenido de: https://docplayer.es/114294520-Introduccion-a-tinkercad.html

Tapia-Ayala \& Manzano-Yupa (2013), Evaluación de la plataforma Arduino e implementación de un sistema de control de posición horizontal. Obtenido de: https://dspace.ups.edu.ec/bitstream/123456789/5522/1/UPS-GT000511.pdf

\section{【Ciencia}




\section{Conciencia}

El artículo que se publica es de exclusiva responsabilidad de los autores y no necesariamente reflejan el pensamiento de la Revista Conciencia Digital.

\section{Ciencia \\ Ligital}

El artículo queda en propiedad de la revista y, por tanto, su publicación parcial y/o total en otro medio tiene que ser autorizado por el director de la Revista Conciencia Digital.


Indexaciones

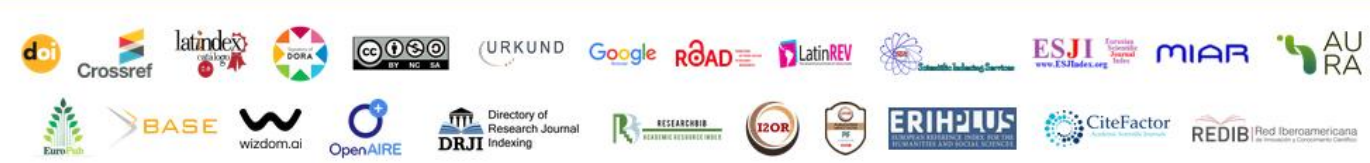

